\title{
Влияние термических и плазменных обработок на фотолюминесценцию пленок оксида цинка
}

\author{
(C) Х.А. Абдуллин ${ }^{1}$, Л.В. Гриценко ${ }^{1,2}$, С.Е. Кумеков ${ }^{2}$, А.А. Мархабаева ${ }^{1}$, Е.И. Теруков ${ }^{3}$ \\ ${ }^{1}$ Национальная нанотехнологическая лаборатория открытого типа (ННЛОТ), \\ Казахский национальный университет им. аль-Фараби, \\ 050000 Алматы, Казахстан \\ ${ }^{2}$ Казахский национальный исследовательский технический университет им. К.И. Сатпаева, \\ 050013 Алматы, Казахстан \\ ${ }^{3}$ Физико-технический институт им. А.Ф. Иоффе Российской академии наук, \\ 194021 Санкт-Петербург, Россия \\ E-mail: aiko_marx@mail.ru
}

(Получена 11 апреля 2017 г. Принята к печати 24 апреля 2017 г.)

Исследованы структура и электрические свойства пленок $\mathrm{ZnO}$, влияние отжига и плазменной обработки в атмосфере водорода на спектры фотолюминесценции пленок ZnO. Кратковременная плазменная обработка в атмосфере водорода приводит к улучшению электрических свойств и появлению интенсивной полосы краевой фотолюминесценции. Наблюдалась сильная зависимость интенсивности и формы спектра краевой фотолюминесценции от условий предварительного отжига. Показано, что спектр фотолюминесценции состоит из нескольких вкладов. Обнаружен эффект инициированного ультрафиолетовым светом увеличения интенсивности фотолюминесценции, который имеет обратимый характер, фотолюминесценция усиливается после ультрафиолетовой засветки и уменьшается до начального значения при хранении образцов в темноте. Обсуждается природа наблюдаемых метастабильных мелких доноров.

DOI: 10.21883/FTP.2018.02.45442.8607

\section{1. Введение}

Оксид цинка является перспективным материалом для применения во многих областях, таких, как светоизлучающие диоды, пьезоэлектрические структуры, тонкопленочные транзисторы [1-3]. Различные сенсоры на основе $\mathrm{ZnO}$ [4-6] благодаря своей реакции на широкий круг веществ весьма перспективны для практических применений и интенсивно исследуются. Оксид цинка обладает яркой фотолюминесценцией при комнатной температуре, что может быть использовано для создания светодиодов и датчиков $[7,8]$. Несмотря на многолетние исследования люминесцентных свойств оксида цинка [9], некоторые вопросы все еще остаются неясными, в частности, природа краевой полосы фотолюминесценции (NBE - near band edge emission) при комнатной температуре, роль поверхностных дефектов и инициированные светом реакции на поверхности.

В настоящей работе проведено исследование влияния отжига и плазменной обработки в атмосфере водорода на спектры фотолюминесценции поликристаллических пленок оксида цинка, на их электрические свойства, обнаружено изменение интенсивности и формы спектра межзонной фотолюминесценции при освещении ультрафиолетовым светом, обнаружены обратимые изменения спектров фотолюминесценции после выдержки образцов под освещением и в темноте.

\section{2. Методика эксперимента}

В работе исследованы высокочистые поликристаллические пленки $\mathrm{ZnO}$, выращенные на стеклянных под- ложках при температуре $\sim 200^{\circ} \mathrm{C}$ методом осаждения металлоорганических соединений из газовой фазы (MOCVD) и легированные бором. Отжиг образцов проводился на воздухе и в различных атмосферах при температуре до $550^{\circ} \mathrm{C}$. Обработку в водородной плазме (Н-обработка) проводили при комнатной температуре в кварцевом трубчатом реакторе диаметром 30 мм. Плазма генерировалась с помощью емкостного возбуждения на частоте 27.12 МГц, мощности около $15 \mathrm{BT}$ и при давлении водорода в реакторе $\sim 50$ Па. Специального нагрева образцов во время обработки не применялось.

Спектры фотолюминесценции (ФЛ) измерялись при комнатной температуре с помощью флуоресцентного спектрофотометра Cary Eclipse (Agilent) при возбуждении импульсной (80 Гц) ксеноновой лампой на длине волны 300 нм. Для модификации спектров ФЛ применялась засветка образцов ультрафиолетовым (УФ) светом с одиночной линией излучения 368 нм от „черной“ УФ лампы детектора купюр мощностью 4 Вт, лампа располагалась на расстоянии $\sim 1$ см от поверхности образца. Одновременно и записью спектров ФЛ определялось электрическое сопротивление пленок при фиксированном напряжении $1 \mathrm{~B}$ с помощью потенциостата Elins P-30J. Электрические свойства (концентрация и подвижность носителей тока, удельное сопротивление) исследованы при комнатной температуре с помощью холловской измерительной системы HMS-3000 (Есоріа). Морфология пленок была изучена с помощью сканирующей электронной микроскопии (SEM) на микроскопе Quanta 3D 200i, кристаллическая структура пленок исследована с помощью рентгеновского дифрактометра (XRD) X'pert PRO MPD. 


\section{3. Экспериментальные результаты}

Типичная морфология поверхности использованных поликристаллических пленок $\mathrm{ZnO}$ характеризуется наличием пирамидных структур со средним размером $100-1000$ нм. Рентгеноструктурные исследования пленок $\mathrm{ZnO}$ демонстрируют преимущественную ориентацию (110) гексагональной структуры вюрцита, о чем свидетельствует доминирование рефлекса при угле дифракции $(2 \theta)$ около $56.6^{\circ}$. Исследованные пленки толщиной 1.2 мкм на стеклянных подложках имеют коэффициент пропускания $70-85 \%$ в интервале длин волн $570-1100$ нм.

Электрические характеристики исходных пленок $\mathrm{ZnO}$ были следующими: концентрация свободных электронов $2.0 \cdot 10^{19} \mathrm{~cm}^{-3}$, подвижность носителей $12 \mathrm{~cm}^{2} / \mathrm{B} \cdot \mathrm{c}$, удельное сопротивление 0.025 Ом · см. Кратковременная (4мин) Н-обработка при комнатной температуре приводила к значениям концентрации, подвижности и удельного сопротивления $7.0 \cdot 10^{18} \mathrm{~cm}^{-3}, 20 \mathrm{~cm}^{2} / \mathrm{B} \cdot \mathrm{c}$ и $0.045 \mathrm{OM} \cdot \mathrm{cm}$ соответственно. Понижение в исходных образцах концентрации мелких доноров после Н-обработки может свидетельствовать о связывании водорода с мелкими донорами с образованием нейтрального комплекса [10].

Отжиг исходных образцов на воздухе в течение часа при $300^{\circ} \mathrm{C}$ приводил к падению концентрации свободных электронов до $4.5 \cdot 10^{18} \mathrm{~cm}^{-3}$, подвижность становилась $5.5 \mathrm{~cm}^{2} / \mathrm{B} \cdot \mathrm{c}$, удельное сопротивление $0.25 \mathrm{OM} \cdot \mathrm{cm}$. Кратковременная (4 мин) Н-обработка при комнатной температуре приводила к восстановлению концентрации, подвижности и удельного сопротивления до значений $1.3 \cdot 10^{19} \mathrm{~cm}^{-3}, 21 \mathrm{~cm}^{2} / \mathrm{B} \cdot \mathrm{c}$ и $0.023 \mathrm{OM} \cdot$ см соответственно. После отжига исходных образцов на воздухе в течение часа при $400^{\circ} \mathrm{C}$ удельное сопротивление вырастало до 100 Ом · см, а Н-обработка приводила к значениям концентрации, подвижности и удельного сопротивления $7 \cdot 10^{18} \mathrm{~cm}^{-3}, 20 \mathrm{~cm}^{2} / \mathrm{B} \cdot \mathrm{c}$ и $0.045 \mathrm{OM} \cdot \mathrm{cm}$ соответственно.

Спектр ФЛ исходных пленок, измеренный при комнатной температуре при возбуждении светом 300 нм, состоит из полосы краевой люминесценции (NBE) с максимумом около 380 нм и полушириной $\sim 0.17$ эВ (рис. 1, кривая 1), а также широкой полосы видимой ФЛ в области 500-750 нм, возникающей благодаря рекомбинации через глубокие уровни (DLE).

Термический отжиг на воздухе в интервале температур от 100 до $300^{\circ} \mathrm{C}$ вызывает небольшой (в $\sim 1.5$ раз) рост интенсивности полосы DLE и значительный (в 10 раз) рост интенсивности полосы $\mathrm{NBE}$, однако при температурах отжига выше $300^{\circ} \mathrm{C}$ интенсивность полосы NBE снова падает. Немонотонное поведение интенсивности полосы NBE при термическом отжиге на воздухе с максимумом при $\sim 300-400^{\circ} \mathrm{C}$ наблюдалось в ряде работ и объяснялось увеличением степени кристалличности [11], десорбцией с поверхности гидроксильных групп [12] и примесных атомов, вызывающих безызлучательную рекомбинацию [13].

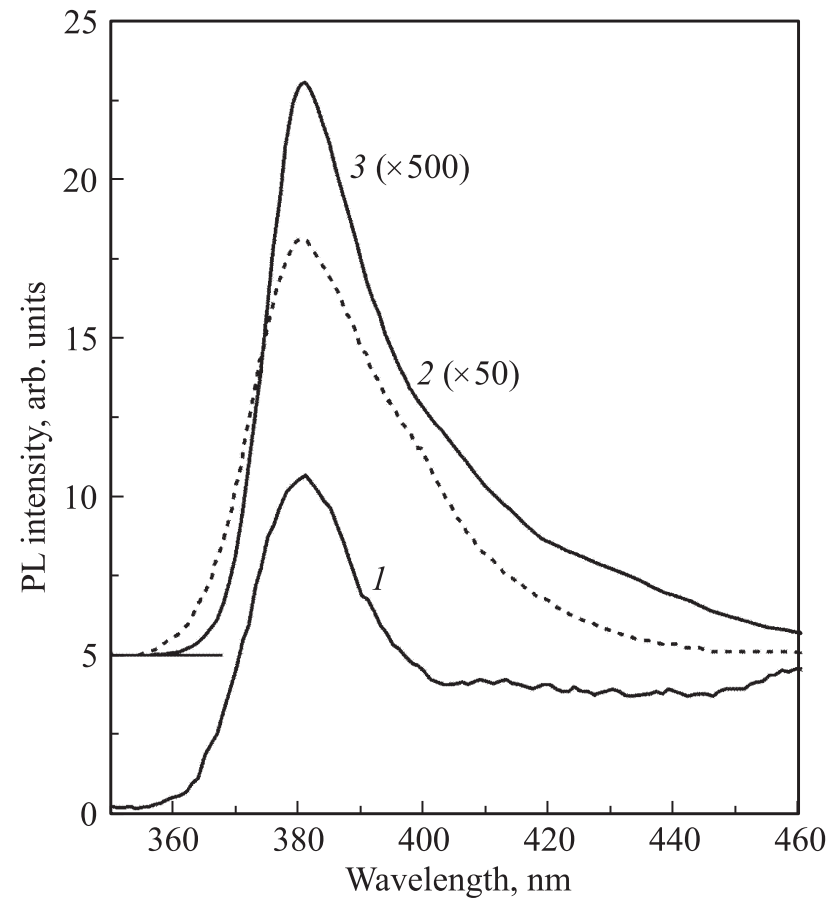

Рис. 1. Спектры ФЛ пленок $\mathrm{ZnO}$, измеренные при комнатной температуре при возбуждении светом 300 нм: 1 - исходный образец, 2 - после плазменной обработки в водороде в течение 4 мин, 3 - отжиг при $400^{\circ} \mathrm{C}$ на воздухе в течение 30 мин с последующей Н-обработкой в течение 4 мин. Для получения истинной интенсивности спектр 2 необходимо увеличить в 50 раз, спектр 3 - в 500 раз.

Обработка $\mathrm{ZnO}$ в водородной плазме приводит к пассивации электрической активности глубоких уровней, о чем свидетельствует рост подвижности носителей. Также наблюдается полное исчезновение полосы DLE излучательных переходов через глубокие уровни в спектрах ФЛ (рис. 1, кривая 2) и рост интенсивности полосы NBE, что согласуется с литературными данными [14-16]. В нашем случае наблюдалось увеличение интенсивности NBE примерно в 50 раз после Н-обработки (рис. 1, кривая 2). Еще больший рост интенсивности полосы NBE наблюдается в образцах, предварительно подвергнутых перед Н-обработкой термическому отжигу на воздухе (рис. 1, кривая 3). Предварительный отжиг в атмосфере азота или в вакууме не дает такого эффекта усиления интенсивности полосы NBE (более подробно эти результаты будут представлены в другой публикации). Оптимальной температурой предварительного 30-минутного отжига на воздухе для достижения максимальной интенсивности NBE после Н-обработки являлся диапазон $350-500^{\circ} \mathrm{C}$. Важно отметить, что увеличение длительности Н-обработки более 15 мин приводило к постепенному падению интенсивности полосы NBE.

Максимум полосы NBE в исходных образцах находился при $3.255 \pm 0.005$ эВ и смещался к $3.280 \pm 0.005$ эВ после Н-обработки, а в образцах, подвергнутых предварительному отжигу при $300-400^{\circ} \mathrm{C}$ на воздухе с 


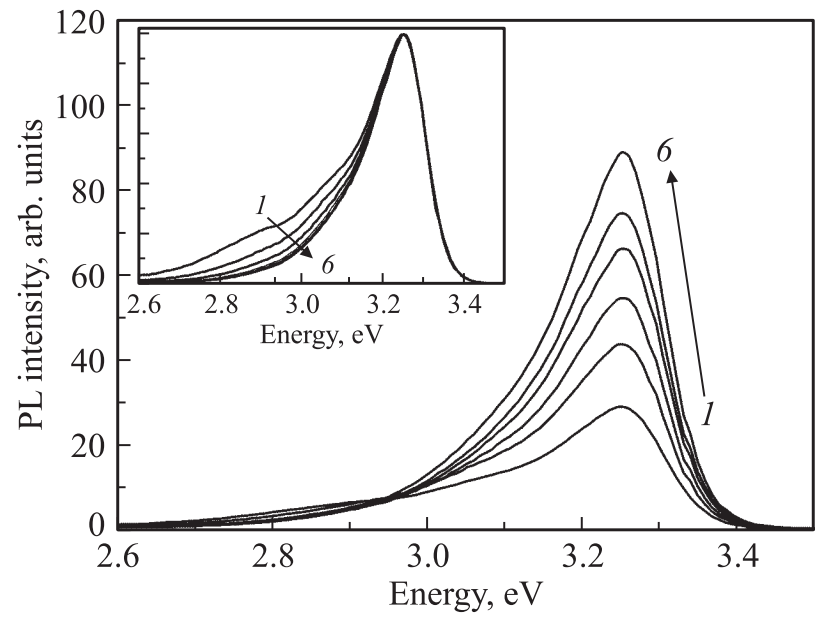

Pис. 2. Спектры ФЛ пленки $\mathrm{ZnO}$, подвергнутой отжигу при $300^{\circ} \mathrm{C}$ на воздухе с последующей обработкой в водородной пламе при комнатной температуре. Спектры сняты при возбуждении при 300 нм при комнатной температуре. Спектр 1 - исходный, спектры 2-6 сняты после засветки образцов УФ-светом с длиной волны 368 нм в течение 8,30 с, 2, 4 и 30 мин соответственно. На вставке - те же спектры, нормированные по максимуму интенсивности.

последующей Н-обработкой, находился при энергии $3.255 \pm 0.005$ эB. Форма линии полосы NBE в спектрах таких образцов также несколько отличается (рис. 1, кривые 2 и 3). Красное смещение полосы NBE на $0.025 \pm 0.005$ эВ в образцах, прошедших предварительный отжиг, и разная форма линии указывают на различные механизмы излучательной рекомбинации в образцах, подвергнутых Н-обработке без отжига либо с предварительным отжигом.

Обнаружено, что возбуждающее люминесценцию излучение с длиной волны от 250 до 360 нм приводит к росту интенсивности полосы NBE. Такое увеличение интенсивности NBE было незначительным, особенно в исходных образцах, из-за низкого уровня интенсивности возбуждающего излучения в спектрометре Cary Eclipse. Поэтому для исследования эффекта УФ-засветки на спектры ФЛ в качестве более мощного источника света была применена „черная“ лампа с длиной волны излучения 368 нм, которая располагалась на расстоянии 1 см от поверхности образца. Засветка проводилась в течение времени от $8 \mathrm{c}$ до 30 мин, затем лампа убиралась, и сразу записывался спектр ФЛ.

Эффект инициированного УФ-светом увеличения интенсивности полосы NBE наблюдался во всех образцах, как исходных, так и подвергнутых обработкам. В исходных образцах интенсивность NBE под действием УФ-засветки росла незначительно, рост мог достигать $5-10 \%$. После отжига на воздухе при $300-400^{\circ} \mathrm{C}$ интенсивность NBE под действием УФ-засветки вырастала уже в $1.4-1.5$ раз. Отжиг на воздухе при $300^{\circ} \mathrm{C}$ с последующей Н-обработкой приводил к росту интенсивности NBE под действием УФ-засветки в $\sim 3$ раза (рис. 2).
Как видно из рисунка, после УФ-засветки наблюдается как рост интенсивности NBE, так и изменение формы линии с уменьшением вклада низкоэнергетической части полосы NBE. Это хорошо видно при нормировании интенсивности спектров к максимуму полосы NBE, как показано на вставке к рис. 2.

Электрическая проводимость пленки $\mathrm{ZnO}$ и интенсивность полосы NBE при облучении УФ-светом растут синхронно и следуют очень близким зависимостям от времени засветки. Поведение проводимости демонстрирует хорошо известный эффект замороженной фотопроводимости, наблюдаемый в $\mathrm{ZnO}$ при УФ-освещении [17-19], природа которого до конца не выяснена.

Интенсивность полосы NBE постепенно уменьшается при хранении образцов в темноте, достигает исходного уровня (т.е. до УФ-засветки) при выдержке в атмосферных условиях в течение нескольких дней, а при повторном измерении снова можно наблюдать эффект увеличения интенсивности ФЛ под УФ-засветкой.

Из рис. 2 видно, что полоса NBE состоит из нескольких линий. Было проведено разложение спектров, приведенных на рис. 2, на гауссовы пики. Результаты аппроксимации двух спектров из рис. 2 (исходного и

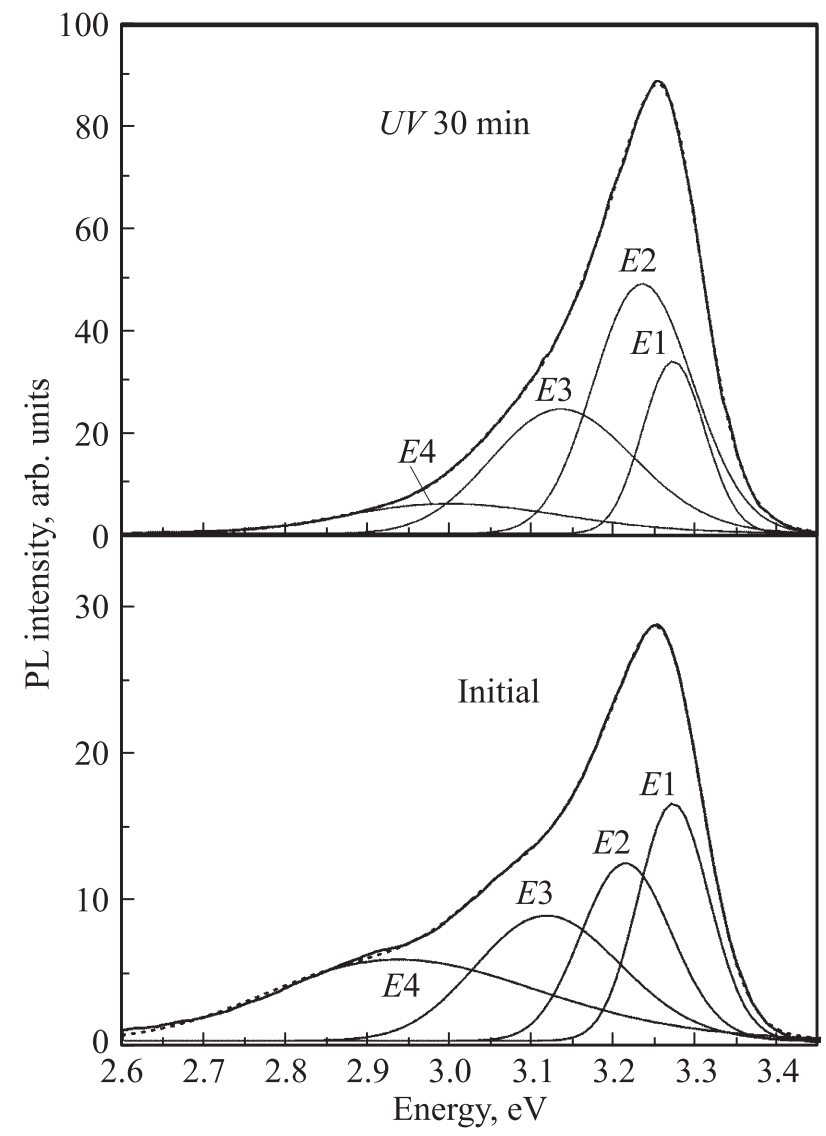

Рис. 3. Сплошными линиями показаны экспериментальные спектры ФЛ из рис. 2 для исходного образца (снизу) и после засветки УФ-светом в течение 30 мин (сверху) и соответствующие разложения на гауссовы пики. Пунктирная линия соответствует сумме четырех гауссовых пиков. 


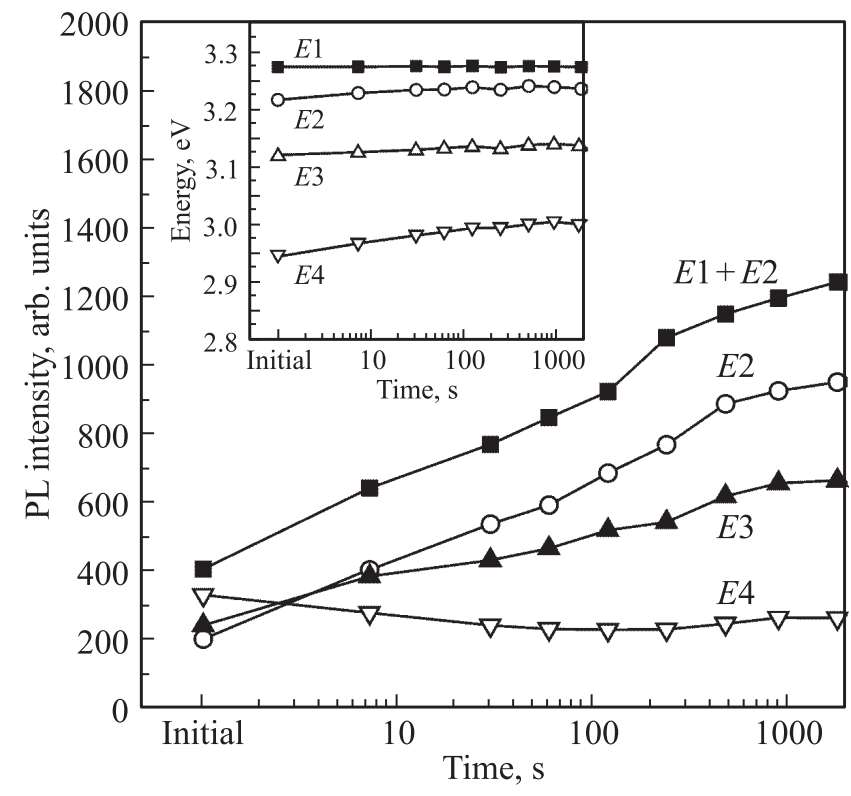

Рис. 4. Интенсивности и энергии (на вставке) гауссовых пиков $E 1-E 4$, аппроксимирующих спектры ФЛ из рис. 2, в зависимости от времени засветки УФ-светом.

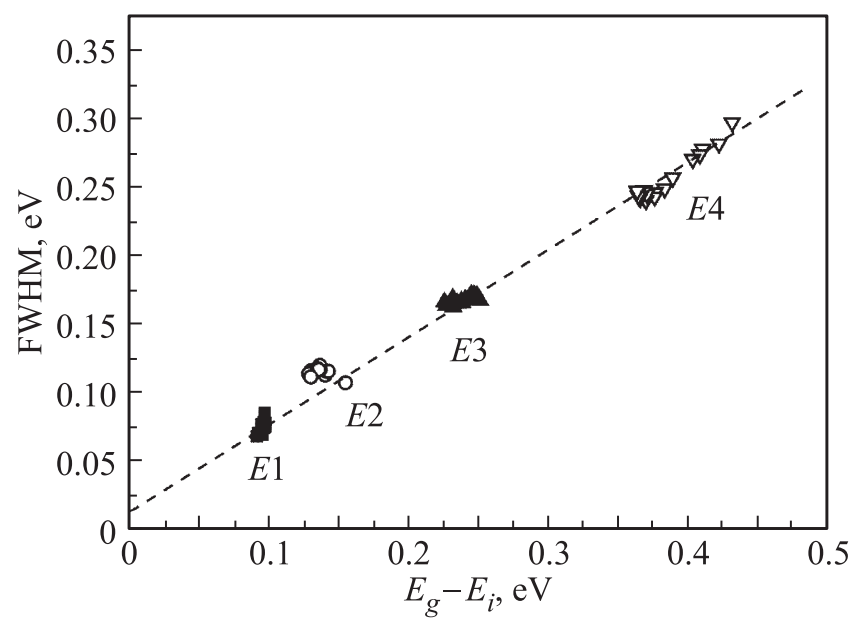

Рис. 5. Зависимости полуширины (FWHM) пиков $E 1-E 4$ от сдвига положения линий относительно края зоны оксида цинка, приведены значения FWHM соответствующих линий во всем интервале времен засветки образцов УФ-светом.

после засветки УФ-светом в течение 30 мин) с помощью гауссовых пиков показаны на рис. 3. Видно, что NBE полоса хорошо разлагается на четыре гауссовых пика, обозначенных как $E 1-E 4$. Исходный спектр (рис. 3 снизу) разлагается на полосы с максимумами при 3.273 эВ (полоса $E 1), 3.215$ эВ $(E 2), 3.119$ эВ $(E 3)$ и 2.938 эВ $(E 4)$, спектр после засветки УФ-светом в течение 30 мин разлагается на полосы $3.273,3.236,3.137$ и 2.999 эВ соответственно. Сходимость аппроксимации спектра четырьмя гауссовыми пиками весьма высокая, аппроксимация практически совпадает с экспериментальными спектрами, полученными для всех длительно- стей УФ-засветки. Заметим, что аппроксимация спектров гауссовыми пиками, сдвинутыми на величину энергии продольного оптического фонона $E_{\mathrm{LO}}=72 \mathrm{MэB}$, дает плохую сходимость.

На рис. 4 показаны зависимости интенсивности гауссовых пиков $E 1-E 4$, аппроксимирующих полученные экспериментальные спектры ФЛ, от времени засветки УФ-светом в интервале от 8 с до 30 мин. Зависимость суммарной интенсивности пиков $E 1-E 4$ от времени УФ-облучения близка к степенной зависимости с показателем $\sim 0.1$. Такая сильно нелинейная зависимость интенсивности NBE от времени УФ-засветки, с большой скоростью изменения в начале и с выходом на насыщение, свидетельствует о поверхностном происхождении эффекта.

На вставке к рис. 4 показано, как энергия пиков $E 1-E 4$ менялась в ходе УФ-засветки. Энергия $E 1$ не меняется во всем интервале времен засветки образцов УФ-светом, а значения $E 2-E 4$ слабо растут. Это говорит о корректности процедуры разложения полосы NBE на 4 гауссовых пика.

Важной характеристикой является полуширина пиков на половине высоты (FWHM). На рис. 5 приведена зависимость FWHM для пиков $E 1-E 4$ от сдвига энергий $E 1-E 4$ относительно края зоны $E_{g}=3.37$ эВ оксида цинка. Величина FWHМ изменяется от $\sim 0.075$ эВ для пика $E 1$ до $\sim 0.25-0.30$ эВ для $E 4$. Как видно из рис. 5, зависимость FWHМ от $E_{g}-E_{i}(i=1-4)$ близка к линейной.

\section{4. Обсуждение результатов}

Экспериментальные результаты показывают, что в исследованных пленках $\mathrm{ZnO}$, как исходных, так и подвергнутых отжигу и Н-обработке, наблюдается эффект инициированного УФ-светом увеличения интенсивности полосы NBE. Насколько нам известно, о таком эффекте в $\mathrm{ZnO}$ ранее не сообщалось, за исключением работы [20], где аналогичный эффект наблюдался в $\mathrm{ZnO}$, выращенном методом осаждения атомных слоев (ALD). Продолжительное освещение $\mathrm{He}-\mathrm{Cd}$-лазером при 5 и $77 \mathrm{~K}$ приводило к увеличению интенсивности полосы NBE, что связывалось с исчезновением рекомбинационных центров на поверхности пленки. В нашем случае рост интенсивности NBE под действием УФ-экспозиции сопровождался синхронным ростом проводимости пленок, поэтому из-за фотодесорбции кислорода может происходить исчезновение электронных ловушек, влияющих на проводимость пленки, и рекомбинационных центров, влияющих на ФЛ.

Снижение темпа рекомбинации на поверхности под УФ-освещением приведет к возрастанию интенсивности ФЛ от областей, расположенных ближе к поверхности. Поэтому интенсивность коротковолновой части полосы NBE будет расти из-за пониженного самопоглощения ФЛ. Однако, если под действием УФ-засветки меняются 
только рекомбинационные условия, интенсивность длинноволновой части полосы NBE не должна уменьшаться в абсолютном выражении. Поскольку уменьшение длинноволновой части полосы NBE весьма заметно (рис. 2), можно сделать вывод, что под действием УФ-засветки меняются не только рекомбинационные условия, но и концентрация центров с мелким уровнем, которые присутствуют в приповерхностной области.

Известно, что в высокочистом $\mathrm{ZnO}$ доминирующий вклад в полосу NBE при комнатной температуре дает фононная реплика $1 L O$ линии свободного экситона $(F X) E_{F X}-1 L O$, максимум полосы находится при $\sim 3.25$ эB [21]. Полоса NBE в $\mathrm{ZnO}$ является асимметричной линией со значительной шириной линии, которая составляет 105-120 мэВ [21,22].

В нашем случае полуширина линии NBE составляла около 167 мэВ, а коротковолновая часть спектра ФЛ хорошо совпадала со спектрами из литературы $[21,22]$, что свидетельствует о наличии дополнительного вклада в форму линии NBE со стороны низких энергий. В исследованных образцах полоса NBE разлагается на 4 гауссова пика, и расхождение между экспериментальным спектром и аппроксимацией практически отсутствует.

Заметим, что УФ-облучение вызывает рост интенсивности пиков $E 1-E 3$ и уменьшение пика $E 4$, обладающего самой низкой энергией. Напротив, выдержка образцов в темноте приводит к падению интенсивности $E 1-E 3$ и росту пика $E 4$, то есть соответствующие центы переходят друг в друга. Поэтому можно предположить, что пики $E 1-E 4$ соответствуют близким по структуре мелким донорам. УФ-облучение вызывает перезарядку центров и переход $E 4 \rightarrow E 3 \rightarrow E 2 \rightarrow E 1$ в состояние с более мелким уровнем. Напротив, в темноте большая часть центров переходит $(E 1 \rightarrow E 2 \rightarrow E 3 \rightarrow E 4)$ в состояние с более глубоким уровнем, и далее в неактивное состояние. Рост энергии доноров от 95 мэВ $(E 1)$ до 380 мэВ $(E 4)$ может происходить за счет взаимодействия с некоторым дефектом (например, акцептором), вызывающим понижение донорного уровня.

Как видно из рис. 5, полуширина пиков $E 1-E 4$ растет с ростом разности между $E_{g}$ и энергией пиков. Если бы пики были фононными повторениями, то следовало бы ожидать, что разность их энергий будет равна энергии оптического фонона, т.е. около $\sim 72$ мэВ, а также что у всех пиков будет примерно одинаковая полуширина FWHM. Этого не наблюдается. Только для пика $E 1$ полуширина составляет $\sim 75$ мэВ, что близко к энергии оптического фонона, для пиков $E 2-E 4$ величина FWHM значительно выше.

Увеличение полуширины пиков по мере сдвига энергии линий $E 1-E 4$ относительно края зоны оксида цинка можно понять, если заметить, что чем меньше энергия связи мелкого уровня, тем сильнее делокализовано его электронное состояние и более эффективно усреднение случайных электрических полей, существующих в приповерхностной области и вызывающих неоднородное уширение линии ФЛ. При росте энергии связи локализация электронного состояния растет, возрастает влияние локальных электрических полей, соответственно растет неоднородное уширение линии ФЛ. Поэтому рост неоднородного уширения линий по мере сдвига положения линий в глубину запрещенной зоны (рис. 5) можно связать с влиянием неоднородных электрических полей.

О природе наблюдаемых центров можно высказать некоторые предположения. Наши результаты свидетельствуют о том, что эффект инициированного УФ-светом увеличения интенсивности полосы NBE особенно ярко проявляется после Н-обработки, поэтому центры $E 1-E 4$ могут быть связаны с водородными донорами. Поскольку эффект многократно усиливается при предварительном отжиге на воздухе, центры могут быть связаны также с дефектами решетки, вводимыми при отжиге в кислородной атмосфере, когда происходит обогащение приповерхностной области, где формируются дефекты $E 1-E 4$, кислородом. Как известно [23], в таких условиях легко образуются вакансии цинка $\left(V_{\mathrm{Zn}}\right)$. При последующей Н-обработке вводимые водородные доноры могут создавать комплексы с дефектами, сформированными в ходе предварительного отжига.

Заметим, что имеются указания на генерацию кислородных вакансий при плазменной обработке в атмосфере водорода. В работе [24] повышение мощности плазменной водородной обработки до 600 Вт приводило к генерации кислородных вакансий, что регистрировалось методом рентгеновской фотоэлектронной спектроскопии. Плазменная обработка при высокой мощности способствовала восстановлению поверхности и созданию избытка атомов цинка, вплоть до появления металлических кластеров на поверхности [24].

Однако в нашем случае кратковременной Н-обработки при низкой мощности плазменного разряда, по-видимому, сохраняется избыток вакансий цинка, сформированных предварительным отжигом на воздухе. Только с увеличением длительности Н-обработки более 15 мин в наших экспериментах происходило постепенное понижение интенсивности NBE полосы, одновременно наблюдалась деградация поверхности образцов, что можно связать с введением кислородных вакансий, созданием избытка атомов цинка и восстановлением поверхности.

О формировании мелких доноров в $\mathrm{ZnO}$ при обработке в водородной атмосфере свидетельствуют результаты как теоретических [23,25], так и экспериментальных работ $[14,15,26]$. Согласно расчетам [23,27], мелким донором в $\mathrm{ZnO}$ является как межузельный водород, связанный с атомом кислорода, так и атом водорода в позиции замещения кислорода $\left(\mathrm{H}_{\mathrm{O}}\right)$. Предполагается, что межузельный водород нестабилен при комнатной температуре и отжигается за несколько недель [28], а водород в позиции замещения стабилен до $\sim 500^{\circ} \mathrm{C}[29,30]$. В спектрах низкотемпературной ФЛ мелкому водородному донору, вводимому Н-обработкой, приписывают полосу $I_{4}[13,31,32]$ при $\sim 3.363$ эВ, которая идентифицирована как водород в кислородной вакансии $\left(\mathrm{H}_{\mathrm{O}}\right)$ [33]. Водородному донору приписывают 
также линию при 3.360 эВ, идентифицированную как мелкий донор - водород в ВС положении [33]. Можно отметить, что обработка $\mathrm{ZnO}$ в водородной плазме часто подавляет узкие пики ФЛ в низкотемпературной полосе NBE, взамен появляется широкая бесструктурная полоса, что, по-видимому, связано с разупорядочением поверхности [32,34]. При этом максимум ФЛ смещается к $\sim 3.36$ эВ. Высокотемпературная (около $900^{\circ} \mathrm{C}$ ) Н-обработка дает узкие линии $I_{4}$ мелких доноров в спектрах низкотемпературной ФЛ [31].

Обнаруженная в настоящей работе структура полосы $\mathrm{NBE}$, состоящая из нескольких пиков, переходящих друг в друга под действием УФ-освещения и в темноте, может принадлежать нескольким близким по природе центрам, которые включают водородный донор, а также дефект либо примесный атом. С учетом увеличения интенсивности ФЛ после отжига на воздухе, эти центры могут включать вакансию цинка. Известно, что вакансии цинка, частично $\left(V_{\mathrm{Zn}} \mathrm{H}\right)$ либо полностью $\left(V_{\mathrm{Zn}_{\mathrm{n}}} \mathrm{H}_{2}\right)$ пассивированные водородом [35], являются акцепторными центрами. Однако дефект, расположенный в приповерхностной области, может кардинально изменять свою электронную структуру по сравнению с дефектом, расположенным в объеме. Кроме того, на поверхности присутствуют адсорбированные атомы, такие, как гидроксильные группы. Можно предположить, что за формирование мелких доноров могут быть ответственны комплексы $3(\mathrm{O}-\mathrm{H})-V_{\mathrm{Zn}}$ и $4(\mathrm{O}-\mathrm{H})-V_{\mathrm{Zn}}[32]$, которые формируются при отжиге и Н-обработке. Появлению таких дефектов может способствовать повышенная концентрация $\mathrm{O}-\mathrm{H}$ связей после отжига в условиях избытка кислорода с последующей Н-обработкой. Выяснение природы наблюдаемых мелких уровней требует дополнительных исследований.

\section{5. Заключение}

Исследованы спектры ФЛ пленок $\mathrm{ZnO}$, полученных методом MOCVD, изучено влияние отжига на воздухе и плазменной обработки в атмосфере водорода на спектры ФЛ. Водородная плазменная обработка приводит к полной пассивации ФЛ через глубокие уровни, как в исходных образцах, так и подвергнутых предварительному отжигу, и появлению интенсивной полосы NBE краевого излучения. Обнаружено, что интенсивность полосы NBE сильно зависит от атмосферы и температуры предварительного отжига.

Обнаружена значительная чувствительность интенсивности полосы NBE к УФ-освещению в интервале длин волн $300-370$ нм. УФ-освещение не только уменьшает поверхностную рекомбинацию, но и вызывает перестройку дефектов, участвующих в создании краевой ФЛ. О наличии метастабильных дефектов свидетельствует рост интенсивности полосы NBE при длинах волн 380 нм и уменьшение интенсивности ФЛ в области $420-430$ нм после УФ-освещения. Эффект инициированного УФ-светом увеличения интенсивности полосы NBE нелинейно зависит от времени УФ-засветки, что свидетельствует об определяющей роли поверхности. Эффект имеет обратимый характер: интенсивность ФЛ возвращается к своему начальному значению при хранении образцов в темноте, но снова увеличивается под УФ-засветкой. Предположено, что наблюдаемые метастабильные дефекты, имеющие мелкий донорный уровень, расположены вблизи поверхности и являются комплексами дефектов с атомами водорода.

\section{Список литературы}

[1] M. Willander, O. Nur, J.R. Sadaf, M.I. Qadir, S. Zaman, A. Zainelabdin, N. Bano, I. Hussain. Materials, 3, 2643 (2010).

[2] A. Kołodziejczak-Radzimska, T. Jesionowski. Materials, 7, 2833 (2014).

[3] S.K. Arya, S. Saha, J.E. Ramirez-Vick, V. Gupta, S. Bhansali, S.P. Singh. Analytica Chimica Acta, 373, 1 (2012).

[4] M.A. Mahjoub, G. Monier. C. Robert-Goumet, F. Réveret, M. Echabaane, D. Chaudanson, M. Petit, L. Bideux, B. Gruzza. J. Phys. Chem. C, 120, 11652 (2016).

[5] R. Kumar, O. Al-Dossary, G. Kumar, A. Umar. Nano-Micro Letters, 7, 97 (2015).

[6] M. Cho, I. Park. J. Sensor Sci. Techn., 25, 103 (2016).

[7] P. Rauwel, M. Salumaa, A. Aasna, A. Galeckas, E. Rauwel. J. Nanomaterials, 2016, Article ID 5320625, 12 pages (2016).

[8] Manoranjan Ghosh, R.S. Ningthoujam, R.K. Vatsa, D. Das, V. Nataraju, S.C. Gadkari, S.K. Gupta, D. Bahadur. J. Appl. Phys., 110, 054309 (2011).

[9] P.A. Rodnyi, I.V. Khodyuk. Opt. Spectroscopy, 111, 776 (2011).

[10] M. Matsubara, M.N. Amini, R. Saniz, D. Lamoen, B. Partoens. Phys. Rev. B, 86, 165207 (2012).

[11] Da-Ren Hang, Sk Emdadul Islam, Krishna Hari Sharma, Shiao-Wei Kuo, Cheng-Zu Zhang, Jun-Jie Wang. Nanoscale Res. Lett., 9, 632 (2014).

[12] Min Young Cho, Min Su Kim, Hyun Young Choi, Kwang Gug Yim, Jae-Young Leem. Bull. Korean Chem. Soc., 32, 880 (2011).

[13] Z.N. Urgessa, J.R. Botha, M.O. Eriksson, C.M. Mbulanga, S.R. Dobson, S.R. Tankio Djiokap, K.F. Karlsson, V. Khranovskyy, R. Yakimova, Per-Olof Holtz. J. Appl. Phys., 116, 123506 (2014).

[14] P.F. Cai, J.B. You, X.W. Zhang, J.J. Dong, X.L. Yang, Z.G. Yin, N.F. Chen. J. Appl. Phys., 105, 083713 (2009).

[15] J.J. Dong, X.W. Zhang, J.B. You, P.F. Cai, Z.G. Yin, Q. An, X.B. Ma, P. Jin, Z.G. Wang, Paul K. Chu. ACS Appl. Mater. Interfaces, 2, 1780 (2010).

[16] A. Dev, R. Niepelt, J.P. Richters, C. Ronning, T. Voss. Nanotechnology, 21, 065709 (2010).

[17] D.A. Melnick. J. Chem. Phys., 26, 1136 (1957).

[18] D. Cammi, C. Ronning. Advances in Condens. Matter Phys., 2014, Article ID 184120, 5 pages (2014).

[19] R. Gurwitz, R. Cohen, I. Shalish. J. Appl. Phys., 115, 033701 (2014).

[20] M.E. Labzowskaya, I.Kh. Akopyan, B.V. Novikov, A.E. Serov, N.G. Filosofov, L.L. Basov, V.E. Drozd, A.A. Lisachenko. Phys. Procedia, 76, 37 (2015).

[21] W. Shan, W. Walukiewicz, J.W. Ager III, K.M. Yu, H.B. Yuan, H.P. Xin, G. Cantwell, J.J. Song. Appl. Phys. Lett., 86, 191911 (2005). 
[22] B. Ullrich, A.K. Singh, M. Bhowmick, P. Barik, D. ArizaFlores, Haowen Xi, J.W. Tomm. AIP Advances, 4, 123001 (2014).

[23] A. Janotti, C.G. Van de Walle. Rep. Prog. Phys., 72, 126501 (2009).

[24] Dong-min Lee, Jae-Kwan Kim, Jinchen Hao, Han-Ki Kim, Jae-Sik Yoon, Ji-Myon Lee. J. Alloys Comp., 583, 535 (2014).

[24] C.G. Van de Walle. Phys. Rev. Lett., 85, 1012 (2000).

[26] Fang-Hsing Wang, Hung-Peng Chang, Chih-Chung Tseng, Chia-Cheng Huang, Han-Wen Liu. Current Appl. Phys., 11, S12 (2011).

[27] A. Janotti, C.G Van de Walle. Nature Materials, 6, 44 (2006).

[28] S.J. Jokela, M.D. McCluskey. Phys. Rev. B, 72, 113201 (2005).

[29] G.A. Shi, M. Saboktakin, M. Stavola, S.J. Pearton. Appl. Phys. Lett., 85, 5601 (2004).

[30] M.D. McCluskey, M.C. Tarun, S.T. Teklemichael. J. Mater. Res., 27, 2190 (2012).

[31] J. Rodrigues, T. Holz, R.F. Allah, D. Gonzalez, T. Ben, M.R. Correia, T. Monteiro, F.M. Costa. Scientific Rep., 5, 10783 (2015).

[32] J.K. Dangbégnon, K. Talla, J.R. Botha. Optical Mater., 34, 920 (2012).

[33] J. Weber, E.V. Lavrov, F. Herklotz. Physica B, 407, 1456 (2012).

[34] Y.M. Strzhemechny, H.L. Mosbacker, D.C. Look, D.C. Reynolds, C.W. Litton, N.Y. Garces, N.C. Giles, L.E. Halliburton, S. Niki, L.J. Brillson. Appl. Phys. Lett., 84, 2545 (2004).

[35] M.G. Wardle, J.P. Goss, P.R. Briddon. Phys. Rev. Lett., 96, 205504 (2006).

Редактор Г.А. Оганесян

\section{Effect of annealing and plasma treatment on the photoluminescence of zinc oxide films}

Kh.A. Abdullin 1, L.V. Gritsenko 1,2, S.E. Kumekov², A.A. Markhabaeva ${ }^{1}$, E.I. Terukov ${ }^{3}$

${ }^{1}$ National Nanotechnology Laboratory of Open Type (NNLOT), Kazakh National University after al-Farabi, 050000 Almaty, Kazakhstan

2 Kazakh National Technical University

after K.I. Satpaev,

050013 Almaty, Kazakhstan

3 loffe Institute,

194021 St. Petersburg, Russia

Abstract The structure and electrical properties of $\mathrm{ZnO}$ films have been investigated. The effect of annealing and hydrogen plasma treatment on the photoluminescence spectra of $\mathrm{ZnO}$ films has been studied. As a result of short-term plasma treatment in hydrogen atmosphere, the electrical properties are significantly improved and an intense near band gap emission appears. A strong dependence of the intensity and shape of the photoluminescence spectrum on the conditions of preliminary annealing was observed. It is shown that the spectrum of near band gap emission consists of several peaks. A significant increase in the intensity of photoluminescence is found upon exposure with UV light, and the intensity of photoluminescence decreases to the initial value for several days if the sample is stored in the dark. The nature of the observed metastable shallow donors and reversible changes in the photoluminescence spectra is discussed. 